\title{
Wireless Controlling Of Remote Electrical Device Using Android Smartphone
}

\author{
Omar Abdulraheem Mahdi ${ }^{1}$, Bhavya Alankar ${ }^{2}$ \\ ${ }^{1,2}$ (Department of Computer science, Faculty of Management Studies and Information Technology/Hamdard \\ University, New Delhi, India)
}

\begin{abstract}
Controlling the home appliances and electronics gadgets wirelessly through SmartPhone technology can be more easy. That is, through this paper, we target to control a light emitting device (LED) from any place in the world and the unique concept within our application is, utilizing the data channels of GSM technology and not the voice channels. In other words, the SmartPhone application's interface will enable the user to control that device using GPRS.The SmartPhone application will connect to an electronic chip(Arduino) to which an LED will be connected. The mode of connection will be GPRS which we have planned to use.
\end{abstract}

Keywords: Smartphone, LED, GPRS, GSM, Arduino.

\section{INTRODUCTION}

The installation of long wires to reach remote places is more expensive than the use of a mobile network that can perform the same task. The Global system for mobile communication (GSM) was initially designed for voice; it can be used to serve other purposes than talking.

GSM can be used as the communication via to receive signals captured by machines in remote places, and also to send control signals to remote machines.[3]

in this paper we have planned to develop an application which would enable the users to display the remote controlling of an electronic device using GSM channel.

The concept of remote controlling is mainly useful when we want to switch ON/OFF an electrical appliance from any geographical location.

\section{Why use a wireless electric controlling System?}

The traditional wired electric control is a great system, but it can be difficult to control the areas of access. Often the range of a wireless network reaches outside the physical boundaries of an organization.[4] The concept of remote controlling is also very prominently used in home security systems, surveillance systems, intrusion detection mechanisms etc.[1]

Also if we have a large organization or building with several electrical appliances installed. Every electrical appliance has its own control switch using which a person can turn ON or OFF the appliance.

Now, if under circumstances, the user needs to turn ON or OFF thee appliances of all or several rooms which might be located far away from each other, it would be little cumbersome. This might be aggravated with the fact that the user needs to control i.e. turn ON or OFF the appliances immediately for some necessary, urgent reason or may be, he/she needs to do that every day, routinely, in order to save electricity bills and the money incurred on their bills.

If the user could conveniently control several electrical appliances by just sitting at one place, whenever needed, under certain circumstances, it would bring a lot of convenience which would also encourage the users to spend and invest the time saved in other constructive tasks. As moving in a large building or a large penthouse or a bungalow could be very time consuming, only to turn ON or OFF the electrical appliances, a one stop solution would definitely pave the way for users to save their time and energy spent on just moving around. Plus, in urgent situations, it is highly essential that instead of manually moving around to control the appliances, the user should be able to do that quickly and sitting at one place. For ex: Suppose, there is a burglar in the premises or some unlawful activity which might be going to happen. If the user has this power to swiftly turn OFF or turn ON all the devices, as the situation might demand, the event could be effectively and proactively avoided by raising an alarm after turning OFF all the appliances.

\section{HARDWARE DESIGN AND INTERFACING}

Arduino is a tool for making computers that can sense and control more of the physical world than your desktop computer. It's an open-source physical computing platform based on a simple microcontroller board, and a development environment for writing software for the board. Arduino can be used to develop interactive objects, taking inputs from a variety of switches or sensors, and controlling a variety of lights, motors, and other physical outputs. Arduino projects can be stand-alone, or they can communicate with software running on your 
computer (e.g. Flash, Processing, MaxMSP). The boards can be assembled by hand or purchased preassembled; the open-source IDE can be downloaded for free. The Arduino programming language is an implementation of Wiring, a similar physical computing platform, which is based on the Processing multimedia programming environment.

\section{Why using Arduino?}

It is flexible, offers a variety of digital and analog inputs, SPI and serial interface and digital and PWM outputs. It is easy to use, connects to computer via USB and communicates using standard serial protocol, runs in standalone mode and as interface connected to PC/Macintosh computers It is inexpensive, around $\$ 30$ per board and comes with free authoring software. It is an open-source project, software/hardware is extremely accessible and very flexible to be customized and extended. Arduino is backed up by a growing online community; lots of sources are already available.

\section{Arduino interfacing with Softwares:}

The Arduino can "talk", (transmit or receive data) via a serial channel, so any other device with serial capabilities can communicate with an Arduino. It doesn't matter what program/programming language is driving the other device. You can either use the Arduino's "main" serial port, the one it uses when you "talk" to it to program it, or you can leave that channel dedicated to programming (and the development environment's serial monitor), and use two other pins for an extra serial link dedicated to the external device. Some programs (like Flash) don't have native serial capabilities. They can still communicate with Arduino through an intermediary which, like a "translator", enables them to talk to each other

\section{Connecting a Switch to the Arduino Board :}

This is probably the simplest possible example to get started with Arduino. It uses an external switch and the Arduino board to turn ON or OFF the on-board LED :

1. Connect a switch (you can replace the switch with a plain jumper wire) to the Arduino board in the following way:

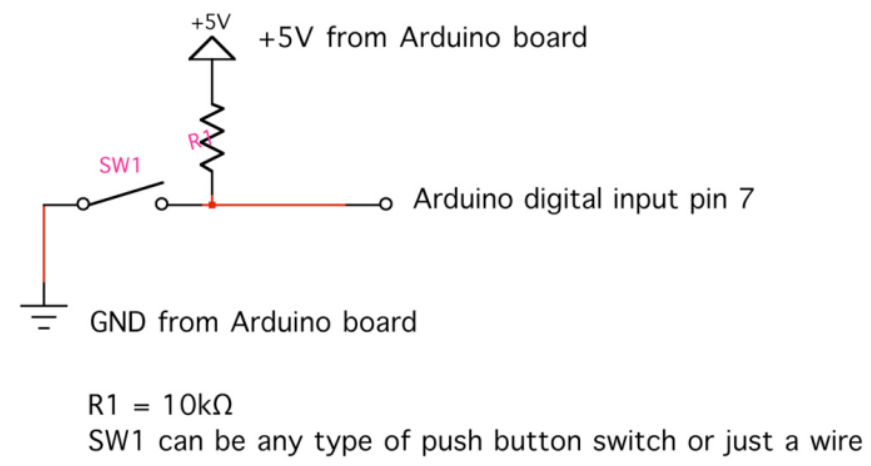

Why do we need the resistor R1? R1 guarantees that the Arduino's digital input pin 7 is connected to a constant voltage of $+5 \mathrm{~V}$ whenever the push button is not pressed. If the push button is pressed, the signal on pin 7 drops to ground (GND), at the same time the Arduino's $+5 \mathrm{~V}$ power is connected to GND, we avoid a shorted circuit by limiting the current that can flow from $+5 \mathrm{~V}$ to GND with a resistor $(1-10 \mathrm{~K} \Omega)$. Also, if there was no connection from pin 7 to $+5 \mathrm{~V}$ at all, the input pin would be "floating" whenever the pushbutton is not pressed. This means that it is connected neither to GND nor to $+5 \mathrm{~V}$, picking up electrostatic noise leading to a false triggering of the input.

2. Open the Arduino software and starting written the codes

3. Download the code into the Arduino board:

a. Select the serial device of the Arduino board from the Tools | Serial Port menu. On the Mac, this should be something with /dev/tty.usbserial in it.

b. Compile your code using the "Verify" button in the top left corner of the software window.

c. Push the reset button on the board then immediately click the Upload button in the environment (don't wait for the board to "come back to life" before pressing the upload button). Wait a few seconds. If successful, the message "Done 
uploading." will appear in the status bar.

d. You are ready to test your first Arduino sensor application.[10]

\section{Microcontroller}

The Arduino Uno is a microcontroller board based on the ATmega328 (datasheet). It has 14 digital input/output pins (of which 6 can be used as PWM outputs), 6 analog inputs, a $16 \mathrm{MHz}$ ceramic resonator, a USB connection, a power jack, an ICSP header, and a reset button. It contains everything needed to support the microcontroller; simply connect it to a computer with a USB cable or power it with a AC-to-DC adapter or battery to get started. The Uno differs from all preceding boards in that it does not use the FTDI USB-to-serial driver chip. Instead, it features the Atmega16U2 (Atmega8U2 up to version R2) programmed as a USB-to-serial converter.[9]

\section{PROPOSED METHODOLOGY}

As we know that the technology is advancing by leaps \& bounds and mobile technology has become an integral necessity in our lives today. We wish to get everything done right from the place where we are. Be it Banking, News, Chatting, Networking, Talking, Locating someone, etc. We cannot imagine certain significant tasks without the mobile technology.

Embedded systems has become a buzz word in the last fifteen years, but embedded systems and processors have been around for much longer than that. Some of the embedded systems are now commonly interfaced with Bluetooth and GSM (Global System for Mobile communication) modules to widen their scope and enhance the application areas to a greater extent.

Although the GSM was initially designed for voice, it can be used to serve other purposes than talking. This idea is reinforced by the fact that the GSM infrastructure has been deployed in many countries. GSM can be used as the communication via to receive signals captured by machines in remote places, and also to send control signals to remote machines.

The installation of long wires to reach remote places (i.e. bridges, vending machines, etc.) is more expensive than the use of a mobile network that can perform the same task. Of course suitable sensors and actuators are needed for the mentioned examples and others. Some automatic GSM module is also needed, but long wired installation is not necessary. Furthermore, mobile telephone can also be used in remote systems, but in moving systems as well, such as vehicles and people. Also Bluetooth is a popular mechanism for short distance point to point or point to multi-point communication. The features include low cost, low power and small size. Also the robustness for the interferences has made Bluetooth a highly versatile and attractive[2]

To further enhance this idea, I have planned to develop a mobile application which would be able to control an electronic device remotely. $i$ have planned to interface the mobile technology with electronics. That is, through this application, we target to control a light emitting device (LED) from any place in the world.

We can easily assume \& correlate this idea with controlling the home appliances in our homes through mobile. That is, how great $\&$ convenient would it be, if we are able to control our home appliances from any part of the world through mobile technology?

In order to develop this application, The mobile application will connect to an electronic chip to which an LED will be connected. The mode of connection will be GPRS which we have planned to use. This project will have two parts namely: Mobile Part and Electronic Part. The mobile part will have an interface through which we would be able to turn ON/OFF the LED remotely from any part of the world.The electronics part will consist of a chip to which an LED will be connected.

We have planned to use GPRS as the medium of information exchange. Once the user presses ON button on the mobile handset, the LED will turn ON or enable. Otherwise, if the user presses OFF, the LED will go off and be disabled. This communication will occur through mounting a shield on the chip which will enable it to obtain an IP that would eventually help the connection between chip \& mobile.

Planned to program the chip in $\mathrm{C} / \mathrm{C}++$ and it will communicate with the mobile application that will be developed in J2ME. This application would be a culmination of two very different technologies which will make it unique \& strong as a whole. . This concept is also very prominently used in home security systems, surveillance systems etc. There would be three parts: Electronics Part, Web Part and Mobile Part.

1. Electronics Module: This module would consist of a small electronic board name Arduino that we would program in C. In addition to many other components, this board consists of a programmable microcontroller, EEPROM, Flash Memory etc.

On the top of this (base) board, we would be mounting an Ethernet shield which would give us the freedom to connect it to a system which would act as the remote server in our project. [5] 
We assume that the remote server would be in an ON state always and connected to internet. Once the electronic board is connected to the remote server, it will receive an IP which our mobile application would be using. We would also connect an LED to this board which can be turned ON/OFF by the mobile application.

2. Web Part: This module would be running at the remote web server end to which the electronic board would be connected via LAN cable. This module would be mainly responsible for processing operations from the electronic board and the commands being received from the remote mobile handset via GPRS.[6]

That is, when the remote mobile handset sends any signal via GPRS to the remote server which is connected to the internet, it will send appropriate instructions or signals to the electronic board Arduino for switching ON/OFF the LED connected to the board.

That is, whatever command the user will give through the mobile application will be appropriately converted to the signal and transmitted to the electronic board. The web module would be developed in J2EE involving JSP, Servlets etc.[7]

This module would not be accepting any user input but would mainly act as the processing stub.

3. Mobile Module: This part would be the actual application that will be developed on J2ME for Android platform. Once this application is developed, its corresponding .jar file would be transferred from the system to the Android mobile device for installation.

Once the application is installed, its mobile user interface will give the user 2 basic options to control the electronic device i.e. ON \& OFF. Once the user pushes the ON button on the mobile user interface, the command will travel via GPRS to the IP of the remote server where the web part is configured.

We will program the mobile application in such a manner that it points only to the IP of the remote server and when the command is send from the mobile application, only the remote web server should attend $\&$ process it.

Furthermore, we also assume that the Android mobile device would be GPRS enabled so that it connect to the internet and use that medium to connect to the remote server from virtually any location, irrespective of the geographical coordinates.[8]

Once the mobile command is received by the remote server, it will convert it to the appropriate electrical signals and send it to the board. In the board, we already have a running, embedded $\mathrm{C}$ program which will understand those signals and will switch ON/OFF the LED as desired and selected by the user from his/her mobile application.

We are also evaluating the possibilities if the remote web part could be eliminated. In that case, only the electronics part would be there with an embedded $\mathrm{C}$ program. Then it will be connected to the internet via Ethernet port and LAN cable to a computer and that board will automatically obtain an IP. We are seeing the possibilities if the mobile application can directly communicate and interface with the electronic board without the involvement of a remote web server.

\section{DIAGRAMMATIC REPRESENTATION}

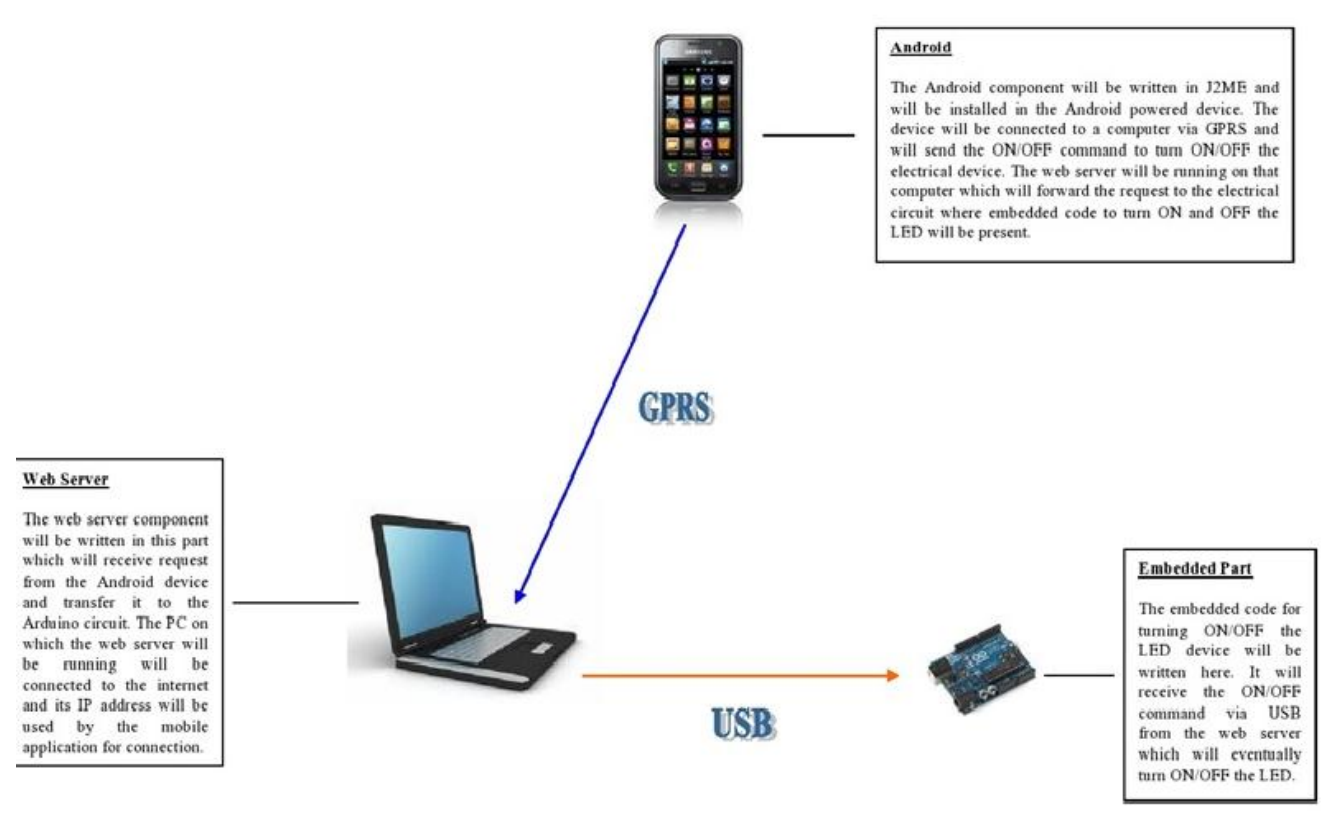




\section{FUTURE SCOPE}

Although the application is developed on a cutting edge technology, it holds a great scope of improvement \& enhancement as mentioned below:

- We can further strengthen this application to control a real time electrical device.

- The instruction from Android handset can check if GPRS feature is enabled. In case, it is not, it can automatically enable it.

- We can further improve this application to use GSM technology for sending the command instructions for switching $\mathrm{ON} / \mathrm{OFF}$ an electrical appliance

- This application can be enhanced to control multiple electrical devices at the same time at different geographical locations

- We can improve this application so that one electrical device could be controlled from multiple locations through different Android handsets

- $\quad$ For different types of electrical devices, we can customize this application for handling different types of operations. For ex: for fans, we can develop a module that controls the speed of the fans also in addition to turning them ON/OFF

- We can build artificial intelligence in this system so that it can be used as a home security systems and does tasks like measuring room temperature and in case of a fire, should turn ON water sprinklers and also inform nearest fire station through Call/SMS or buzzer alarms

\section{CONCLUSION}

- This application would enable a user to control an electrical device effectively using GPRS technology

- This application will empower the users to save time and energy

- Since it involves GPRS, the user would be able to control the device from a geographically independent location

- It would facilitate the home-based security applications through remote controlling of any electrical device

- This application can also be used to measure room temperature \& humidity with some minor additional hardware.

\section{REFERENCES}

[1] Prakash Kumar, Pradeep Kumar, Arduino Based Wireless Intrusion Detection Using IR Sensor and GSM,, International Journal of Computer Science and Mobile Computing, 2(5), 2013, 417-424.

[2] Vini Madan , S.R.N. Reddy, "GSM-Bluetooth based Remote Monitoring and Control System with automatic Light Controller", International Journal of Computer applications ,46(1), 2012, 20-28.

[3] G. Aranguren, L. Nozal, A. Blazquez, and J. Arias, "Remote control of Sensors and actuators by GSM", IEEE 2002 28th Annual Conference of The Industrial Electronics Society IECON 02, vol. 3, 2002, pp.2306 - 2310.

[4] Arnaud Henry-Labordere, Virtual Roaming Systems for GSM, GPRS and UMTS (John Wiley \& Sons Ltd, 2009).

[5] John Boxall, Arduino Workshop: A Hands-On Introduction With 65 Projects (Copy Right Materiel, 2013).

[6] .Christoffer Andersson, GPRS and $3 G$ Wireless Applications(Professional Developer's Guide, 2009)

[7] Mahesh P. Matha, JSP AND SERVLETS: a Comprehensive Study (PHI Learning Private Limited, 2013)

[8] Lauren Darcey and Shane Conder, Android Wireless Application Development (developer's Library, 2012)

[9] Jayanta Kumar Pandey, R.N. Das choudhary, "Embedded Automobile Engine locking System, using GSM technology", ITER, SOA University Odisha, India.

[10] Fabian Winkler,"Arduino workshop", spring 2007, http://www.arduino.cc . 